\title{
Alfabetización académica y competencia comunicativa en educación superior*
}

\author{
Juan Antonio Núñez Cortés \\ Universidad Autónoma de Madrid / \\ Universidad CEU San Pablo
}

U

\section{Resumen}

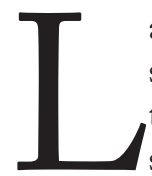

a lectura y la escritura no solo son herramientas para transmitir información sino que son procesos cognitivos y socioculturales que favorecen la adquisición de conocimientos en cualquiera de los niveles educativos en los que se desarrolle. En este sentido, el presente artículo realza la importancia de la lectura y la escritura en la universidad y se relaciona con la alfabetización académica y la competencia comunicativa. Asimismo, se hace una revisión de cómo se ha atendido a estos dos conceptos en el ámbito iberoamericano y, también, desde organismos internacionales como la Unión Europea y la UNESCO. Por último, se resalta la idea de continuar realizando investigaciones sobre la cuestión y fomentar políticas de alfabetización académica. Este texto es fruto de la conferencia inaugural impartida en el marco de las IV Jornada de Innovación Educativa "Estudiantes del siglo XXI: aprendizaje, competencias e innovación" de la Universidad Nacional Autónoma de Honduras. Brevemente, se responden dos preguntas que coinciden con los dos apartados del artículo: ¿por qué la alfabetización académica? y ¿qué atención se da a la competencia comunicativa desde los organismos internacionales en la educación superior?
Palabras clave: Alfabetización académica, competencia comunicativa, leer, escribir.

\section{Abstract}

Reading and writing are not only some kind of tools used to transfer information, but are also cognitive and socio-cultural processes which contribute to the acquisition of knowledge in any educational level in which they may develop. In this sense, the aim of this article is to drawn the attention to the importance of the reading and writing in the university and its relation with the academic literacy and communicative competence. Furthermore, it makes a review of how the Latin-American community has seized these two concepts, and also how it was done by the international bodies, such as the European Union and UNESCO. Finally, it highlights the need to continue carrying out investigations in this field and encourage the academic literacy politics. This material is the result of the inaugural conference provided during the IV Day of Educational Innovation "Students of the XXI century: learning, competences and innovation" of the Autonomous National University of Honduras. Briefly, it responds to two questions which correspond to the two sections of the article: "why the academic literacy?" and "what significance is given by the international bodies in the higher education to the communicative competence?".

Key words: Academic literacy, communicative competece, reading, writing

\section{¿Por qué la alfabetización} académica?

En la sociedad globalizada del conocimiento o del aprendizaje, fluctuante y digital, las personas han de tener la capacidad de adaptarse a un cambio continuo. De esta manera -entre muchas otras-, el desempeño en la lectura y la escritura se erige como una competencia clave para el aprendizaje en la educación superior. Así, el desarrollo de la competencia comunicativa en la universidad del siglo XXI tiene que favorecer que los estudiantes universitarios, protagonistas de un nuevo paradigma de enseñanza-aprendizaje, conjuguen información, conocimiento y producción del mismo. Esto en una era de nuevas tecnologías de la información y de la comunicación, en donde la obsolescencia y el avance del saber parecen aliarse, y la internacionalización de la universidad es evidente.

${ }^{\star}$ El contenido esencial de este trabajo se basa en la tesis doctoral del autor que aparece referenciada en las referencias documentales. 
Pero, con independencia de que la competencia comunicativa sea fundamental en la educación superior y en cualquier ámbito de la vida, ¿es necesario enseñar a leer y a escribir en la universidad? ¿Acaso este cometido no es responsabilidad de los niveles previos? Pues bien, pese a que es común la idea de que los estudiantes ingresan en los estudios superiores con un déficit en esta competencia, los problemas y dificultades de comunicación que tienen y a los que se enfrentan no solo se deben a estas carencias. Como señalan Miguel de Cervantes y la nobel Wislawa Szymborska, y parafraseándolos, en la universidad -negro sobre blanco, rigen otras leyes-, es decir, los textos que leen y escriben los estudiantes tienen características particulares que difieren, en ocasiones, de aquellos con los que ya estaban familiarizados $(\mathrm{Pa}-$ rodi, Ibáñez, Venegas y González, 2010). Efectivamente, las formas de comunicación de las comunidades discursivas académicas son singulares (Bazerman, 1998) y los estudiantes no están experimentados con ellas, por lo que encuentran, en ocasiones, una dificultad que les parece imposible.

La necesidad de fomentar iniciativas que favorezcan la adquisición de estos géneros discursivos académicos podría refutarse con la idea de que los estudiantes han de ser capaces de poner en marcha estrategias para saber aprender, en este caso, los géneros académicos propios de las disciplinas de las que ahora comienzan a formar parte. Sin embargo, la lectura y la escritura son herramientas de uso cotidiano en la universidad (Carlino, 2005); y, en concreto, la escritura -además de la registradora- tiene una potente función epistémica, que se pone al servicio del apren-

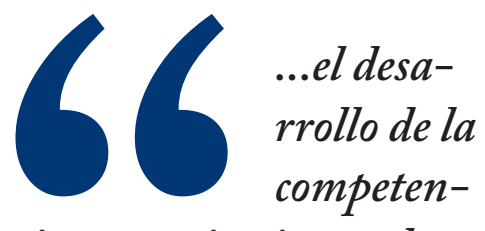

cia comunicativa en la universidad del siglo XXI tiene que favorecer que los estudiantes universitarios, protagonistas de un nuevo paradigma de enseñanza-aprendizaje, conjuguen información, conocimiento y producción del mismo...

dizaje de las disciplinas (Cassany, 1989; Olson 2009; Ong, 1987; Rogers y Walling, 2011; Scardamalia y Bereiter, 1992). Escribir es tomar continuamente decisiones y, por tanto, reflexionar; y es a través de ese proceso de reflexión como la escritura contribuye al aprendizaje. En la reflexión previa al proceso y durante este se resignifican los conoci- mientos (González-Moreno, 2012). En otras palabras, Sartre lo explicita de la siguiente manera: escribir [...] era tomar las cosas, vivas, en la trampa de las frases: si combinaba ingeniosamente las palabras, el objeto se enredaba en los signos y lo tenía. Y la posesión de este objeto, del conocimiento, solo conlleva la adquisición de una voz propia. ¿Cómo, entonces ahora, no contribuir para que desde la universidad los estudiantes adquieran esta voz, su opinión sobre lo que les rodea y estudian? ¿No hará esto que puedan comunicarla y de esta manera participar en la sociedad? Y si leer se entiende como una pregunta al texto de un emisor que escribe desde un contexto siempre con una intención determinada, ¿̇se puede dejar de atender a estrategias de lectura y escritura que desarrollen el pensamiento crítico?

Estas preguntas, por obvias que parezcan, solo han dado lugar, en ocasiones, a respuestas que no han motivado acciones al respecto. Por contra, aquellos que están a favor de la alfabetización académica -entendida esta, como "proceso de enseñanza que puede (o no) ponerse en marcha para favorecer el acceso de los estudiantes a las diferentes culturas escritas de las disciplinas" (Carlino, 2013: 370) sí toman partido a favor.

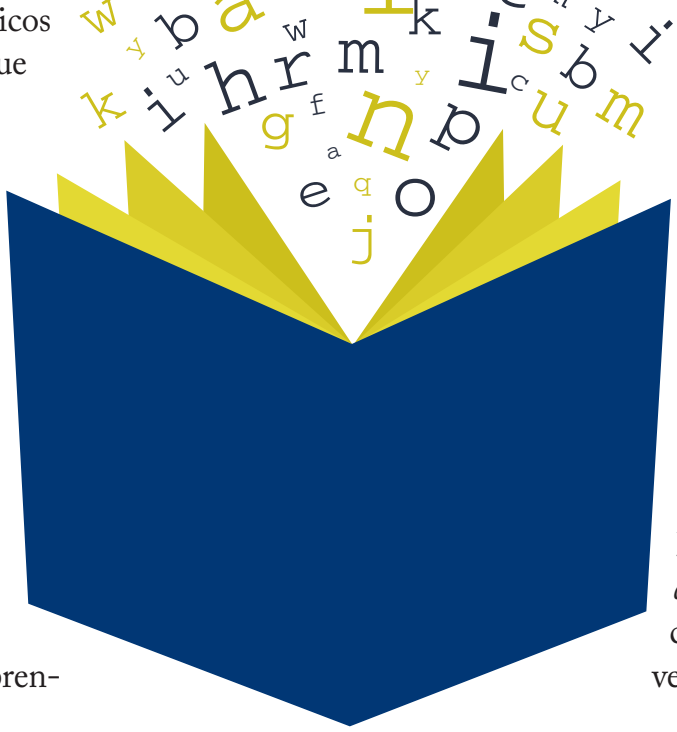

En este sentido, desde el ámbito anglosajón, la atención a la lectura y a la escritura, con base en algunas de las premisas de la alfabetización académica, se viene dando durante todo el siglo XX en Reino Unido y Estados Unidos. En este segundo país, por ejemplo, en la década de los setenta del pasado siglo proliferaron los centros de escritura (writing centers) en las universidades como consecuencia de las políticas universitarias democratizadoras para fo-

1. Según el Academic Ranking of World Universities (2012) de Shanghai Jiao Tong University 
mentar el ingreso de estudiantes con rentas bajas, entre otros (Bazerman y Russell, 1994; Waller, 2002). Al vincular las rentas bajas con el rendimiento educativo, se podría considerar que los centros de escritura tenían una función más remedial de aquel déficit señalado que otras por las que aboga la alfabetización académica como proceso. Lejos de tener solo esta función, en la actualidad, a modo de ejemplo, las veinte universidades más valoradas del mundo en el año 2012 tienen centros de escritura para todos sus estudiantes ${ }^{1}$. De tal manera, en la actualidad, existen asociaciones internacionales y nacionales de centros de escritura, como la internacional (International Writing Centers Association) que engloba, entre otras, a la europea, la africana, la norteamericana y la japonesa.

Esta función remedial de los centros de escritura o de cualquier programa de lectura y escritura en la universi-

dad tendría entre sus iniciativas, por ejemplo, las asignaturas de escritura académica obligatorias al comienzo de las carreras. Frente a este modelo, otro transversal propondría otras iniciativas, como las asignaturas de escritura intensiva en las disciplinas o las tutorías en los centros, que también son impulsadas en muchas ocasiones por dichos centros de escritura (Carlino, 2005a; Núñez Cortés, 2013a). Todas estas acciones, como se ha dicho, son llevadas a cabo en muchas de las universidades anglosajonas.

En el ámbito iberoamericano, la atención a la lectura y a la escritura en la universidad ha aumentado considerablemente a comienzos de este siglo, aunque esta comenzó a gestarse en algunas universidades en la década de los 80 a través, por ejemplo, de talleres de escritura e investigaciones en torno al asunto.

Fruto de este interés, han prolifera- do las investigaciones en América Latina sobre las dificultades de escritura y de lectura de los estudiantes, sus procesos de composición, las estrategias que llevan a cabo cuando leen y escriben, los géneros discursos académicos, las prácticas docentes, así como aquellas que daban cuenta de iniciativas novedosas llevadas a cabo en países que no son iberoamericanos (Arnoux et al, 1996; Arnoux, Nogueira y Silvestri, 2006; Carlino, 2005b, 2010; Cervantes y Rodríguez, 2012; Cortés, Rivera y Rodríguez, 2008; Desinano, 2009; Ilich y Morales, 2004; Martínez, 2010; Romero, 2000). También hay,

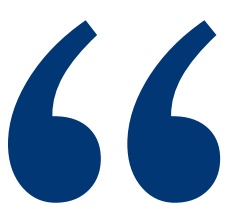

...Escribires tomar continuamente decisiones $y$, por tanto, reflexionar; y es a través de ese proceso de reflexión como la escritura contribuye al aprendizaje...

aunque son escasos, algunos estudios relevantes centrados en los países iberoamericanos, que reflejan cómo estas iniciativas son consecuencia de políticas educativas concretas de alfabetización académica en las universidades, e incluso en los países (Carlino, 2012).

\section{Competencia comunicativa} en educación superior desde la perspectiva de los organismos internacionales

En este marco de la alfabetización académica, al tener ésta como objetivo desarrollar la competencia comunicativa de los estudiantes universitarios, se ve oportuno atender al tipo de atención mostrada a esta competencia por diferentes instituciones desde la perspectiva del modelo competencial. Así pues, este modelo de enseñanza-aprendizaje ha sido adoptado por instituciones de educación superior para dar respuesta a los desafíos que, hoy día, tiene la universidad. Al respecto, es notorio, sin duda, el impulso dado al mismo en el Espacio Europeo de Educación Superior (EEES), pese a que, por ejemplo, el polisémico término de competencia siga sujeto a una constante reinterpretación (Bolívar, 2008; Gimeno Sacristán, 2008). Sin dejar de atender al objetivo de la Unión Europea de ser la economía basada en el conocimiento más competitiva y dinámica del mundo, el interés por fomentar la movilidad y el empleo de los estudiantes universitarios ha favorecido la creación de un marco competencial para la educación superior. En dicho marco, consensuado a partir de los llamados descriptores de Dublín, se han estipulado niveles de desempeño de las competencias, entre las que se encuentra la comunicativa (JQI, 2004; Ministry os Science, Technology and Innovation, 2005).

Asimismo, la tendencia educativa constatada de evaluar la calidad de los sistemas educativos nacionales a través de pruebas de rendimiento de competencias a sus estudiantes durante la educación obligatoria se va filtrando en la educación superior. De esta manera, la Organización para la Cooperación y el Desarrollo Económico (OCDE), en la actualidad, realiza un estudio de viabilidad de evaluación de competencias -entre ellas, de nuevo, la comunicativaa estudiantes universitarios de diferentes países, llamado Assessment of Higher Education Learning Outcomes (AHELO), en la línea de otros estudios como el Informe PISA (AHELO, 2012). 
Al respecto, en el ámbito iberoamericano, las referencias al modelo competencial han sido intermitentes en las últimas dos décadas y en su mayor parte han estado vinculadas al mundo laboral o al compromiso de aspirar a la calidad de la educación obligatoria. Pese a que la Organización de Estados Iberoamericanos (OEI) ha mostrado interés en esta cuestión, como se aprecia en las Metas Educativas 2021, las conclusiones que se vislumbran de las cumbres de los Jefes de Estado y de Gobierno podrían ser interpretadas como una declaración de intenciones a favor del enfoque competencial sin más (Núñez Cortés, 2013b). No obstante, en los últimos años, en la educación superior se ha mostrado más atención al mismo debido al interés manifiesto de crear el Espacio Común de Educación Superior ALCUE y el Espacio Iberoamericano del Conocimiento.

En este orden, la Organización de las Naciones Unidas para la Educación, la Ciencia y la Cultura (UNESCO), pese a que su interés en el tema no haya sido tan acusado, en las dos últimas conferencias mundiales de la educación superior, celebradas en 1998 y 2009, ha hecho referencia a la importancia de desarrollar las competencias en los estudiantes universitarios (Unesco, 2008, 2010). Esto puede corroborar el hecho de que en el propio EEES se entienda que el modelo competencial puede, cuando menos, dar respuestas a lo que la internacionalización de la universidad lleva parejo: la movilidad de los estudiantes, la coherencia en la evaluación de los mismos y la homologación de títulos. En estrecha relación con esta movilidad de estudiantes, pero en general con la movilidad de los ciudadanos de la Unión Europea, desde la política multilingüe europea, el Marco Común Europeo de Referencia para las
Lenguas (Instituto Cervantes, 2002) ha conceptualizado pormenorizadamente la competencia comunicativa y ha establecido niveles de desempeño de la misma; si bien, este documento está destinado a la enseñanza de segundas lenguas.

Con relación a esto, desde diferentes organismos internacionales también se ha mostrado interés por su conceptualización, sus niveles y los aspectos que comporta, pues se considera fundamental para poder conocer el grado de desempeño que tienen los ciudadanos de la misma en los diferentes proyectos e investigaciones que han atendido a la cuestión. En estos programas y estudios se puede apreciar que existe una gran diversidad de propuestas. Estas divergencias aumentan cuando se comparan las propuestas sobre educación universitaria con las que atañen a las específicas de la educación permanente, por paradójico que parezca, puesto que es común considerar que una engloba a la otra. Sea como fuere, los datos sobre el grado de dominio de la competencia comunicativa en personas adultas son muy llamativos, como muestra el estudio ALL de la OCDE. Además, en otros estudios realizados en el ámbito iberoamericano como Tuning América Latina o $6 \times 4$ de la Oficina Regional de Educación de la Unesco para América Latina y El Caribe, Orealc, se desprende la idea de la importancia que se da a la competencia comunicativa no solo por parte de estudiantes y profesores universitarios, es decir, de la comunidad académica; sino también por parte de los trabajadores recién graduados y de los empleadores.

\section{Conclusión}

Con todo, a la luz de lo expuesto, se pueden afirmar dos ideas relevantes que impulsarían investigaciones en torno al desarrollo de la competencia comunicativa y de la alfabetización académica en la universidad. Por un lado, existe acuerdo en considerar a esta competencia como una importante en la universidad y en el mundo laboral, aunque los estudiantes sientan que no han sido formados suficientemente durante sus estudios superiores para el desarrollo de dicha competencia. Por otro, las características propias de la universidad del siglo XXI como la internacionalización, y lo que esta comporta en tanto a movilidad de estudiantes hacia el extranjero y la necesidad de aprender nuevas lenguas en el nuevo contexto, así como la formación y preparación de los estudiantes universitarios para desenvolverse en el ámbito profesional y la evaluación por competencias, habrían de situar a la competencia comunicativa en una posición privilegiada para ser objeto de atención por parte de las instituciones de educación superior.

\section{Referencias}

AHELO (2012). Assessment of Higher Education Learning Outcomes. Recuperado de: http://www.oecd. org/edu/skills-beyond-school/ AHELO\%20Brochure.pdf

Arnoux, E., Alvarado, M., Balmayor, E., Di Stefano, M., Pereira, C. y Silvestri, A. (1996). El aprendizaje de la escritura en el ciclo superior. En E. Arnoux (Comp.), Adquisición de la escritura. Rosario: Juglaría.

Arnoux, E., Nogueira, S. y Silvestri, A. (2006). Lecturas y reescrituras de un texto teórico en estudiantes de profesorado de enseñanza primaria. Signo y Seña, 16, 137-165.

Bazerman, C. (1988). Shaping written knowledge. Madison: The University of Wisconsin Press.

Bazerman, C. y Russell, D. (Eds.). (1994). Landmark Essays on Writing Across the Curriculum. Davis: Hermagoras Press. 
Bolívar, A. (2008). El discurso de las competencias en España: educación básica y edu-cación superior. Revista de Docencia Universitaria 2, 1-23.

Carlino, P. (2005a). La escritura en el nivel superior. La Gaceta, 418, 18-21.

(2005b). Representaciones sobre la escritura y formas de enseñarla en universidades de América del Norte. Revista de Educación, 336, 143-168.

(2010). Estudiar, escribir y aprender en universidades australianas. Textura, 6(9), 11-33.

(2012). Who Takes Care of Writing in Latin American and Spanish Universities? En S. H. McLeod (Ed. de la serie), Perspectives on Writings: Vol. Writing Programs Worldwide: Profiles of Academic Writing in Many Places (pp. 485-

588). Fort Collins, CO: The WAC Clearinghouse-Parlor Press.

(2013). La alfabetización académica diez años después. Revista Mexicana de Investigación Educativa, 18(57), 355-381.

Cassany, D. (1989). Describir el escribir. Cómo se aprende a escribir. Barcelona: Paidós.

Cervantes, M. de (2004). Don Quijote de la Mancha. Madrid: RAE. Cervantes, G. y Rodríguez, A. (2012). Problemas léxico-semánticos en la formulación de textos académicos. Chemins actuels, 73 .

Cortés, J., Rivera, V. M. y Rodríguez, C. M. (2008). Dificultades en la escritura en la Educación Superior. Bogotá: Edisoma.

Desinano, N. B. (2009). Los alumnos universitarios y la escritura académica. Análisis de un problema. Rosario:
Homo Sapiens Ediciones.

Gimeno Sacristán, J. (Comp.), Pérez, A. I., Martínez, J. B., Torres, I., Angulo, J. F. y Álvarez, J. M. (2008). Educar por competencias, ¿qué hay de nuevo? Madrid: Morata.

González Moreyra, R. (1998). Comprensión lectora en estudiantes universitarios iniciales. Persona, 1, 43-65.

Ilich, E. y Morales, O. A. (2004). Análisis de textos expositivos producidos por estudiantes universitarios desde la perspectiva lingüística discursiva.

Educere: Revista Venezolana de Educación, 26, 333-345.

Instituto Cervantes (2002). Marco común europeo de referencia para las lenguas: aprendizaje, enseñanza, evaluación. Madrid: Ministerio de Educación, Cultura y Deporte.

Martínez, J. (2010). La planificación textual y el mejoramiento de la escritura académica. Revista Infancias Imágenes, 9(2), 35-47.

Núñez Cortés, J. A. (2013). Una aproximación a los centros de escritura en Iberoamérica. Legenda, 17(17), 64-102.

(2013). La alfabetización académica: estudio comparado en el ámbito latinoamericano (Tesis doctoral inédita). Madrid: Universidad Autónoma de Madrid.

Olson, D. (2009). Education and literacy. Infancia y Aprendizaje, 32(2), 141-151.

Ong, W. J. (1987). Oralidad y escritura. Tecnologías de la palabra. Buenos Aires: Fondo de Cultura Económica.

Parodi, G., Ibáñez, R., Venegas, R. y González, C. (2010). Identificación de géneros académicos y géneros profesionales: Principios teóricos y propuesta metodológica. En G. Parodi (Ed.), Alfabetización académica y profesional en el siglo XXI. Leer y escribir desde las disciplinas (pp. 249-290). Santiago: Ariel.

Rogers, P. M. y Walling, O. (2011). Writing and Knowledge Making: Insights from an Historical Perspective. En S. H. McLeod (Ed. de la serie), Perspectives on Writings: Vol. Writing in Knowledge Societies (pp. 259-273). Fort Collins, CO: The WAC Clearinghouse-Parlor Press.

Romero, F. (2000). La escritura en los universitarios. Ciencias Humanas, 21.

Sartre, J. P. (s. f.). Las palabras. Recuperado de: http://www. gutenscape.com/documentos/ 1 f04b637-555e-4d53-b07b-

1d53dd- ce11e3.pdf

Scardamalia, M. y Bereiter, C. (1992). Dos modelos explicativos de los procesos de composición escrita. Infancia y Aprendizaje, 58, 43-64.

Szymborska, W. (2005). Paisaje con grano de arena. Badalona: Lumen.

UNESCO (1975). (1998). Conferencia Mundial sobre la Educación Superior. La educación superior en el siglo XXI: Visión y acción.

(2010). Conferencia Mundial de la Educación Superior 2009. París: UNESCO.

Waller, S. C. (2002). A Brief History of University Writing Centers: Variety and Diversity. Recuperado de: http://www.newfoundations.com/ History/WritingCtr.html $\because$ 